acceleration, pressure (for example, during flight or when swimming at depth) and temperature. Such work promises to illuminate not only predator-prey interactions, but also how wild animals cope with other real-world issues $^{9,10}$. For example, this type of research could enhance our understanding of how animals are dealing with the impacts of climate change, or offer insight into the factors governing behaviours such as habitat selection, mating and foraging. Moreover, understanding how animals move might inspire the design of robots that can negotiate complex environments. -

Andrew A. Biewener is in the Department of Organismic and Evolutionary Biology, Harvard University, Massachusetts 01730, USA.

e-mail:abiewener@oeb.harvard.edu

1. Wilson, A. M. et al. Nature 554, 183-188 (2018).

2. Hawkes, L. A. et al. Proc. Natl Acad. Sci. USA 108, 9516-9519 (2011).

3. Portugal, S. J. et al. Nature 505, 399-402 (2014).

4. Tsoar, A. et al. Proc. Natl Acad. Sci. USA 108,
E718-E724 (2011).

5. Wittemyer, G., Getz, W. M., Vollrath, F. \& Douglas-Hamilton, I. Behav. Ecol. Sociobiol. 61, 1919-1931 (2007).

6. Nagy, M., Ákos, Z., Biro, D. \& Vicsek, T. Nature 464, 890-893 (2010).

7. Schaller, G. B. The Serengeti Lion: A Study of Predator-Prey Relations (Univ. Chicago Press, 1972).

8. Moore, T. Y., Cooper, K. L., Biewener, A. A. \& Vasudevan, R. Nature Commun. 8, 440 (2017).

9. Hussey, N. E. et al. Science 348, 1255642 (2015).

10.Kays, R., Crofoot, M. C., Jetz, W. \& Wikelski, M. Science $\mathbf{3 4 8}$, aaa2478 (2015).

This article was published online on 29 January 2018.

\title{
A chirp, a roar and a whisper
}

\section{In 2017, gravitational waves and electromagnetic radiation were detected from the merger of two stellar remnants called neutron stars. An observational analysis reveals how this radiation was released from the merger. SEE LETTER P.207}

\section{RALPH WIJERS}

$\mathrm{L}$ ast year, scientists reported the coalescence of two astronomical objects known as neutron stars ${ }^{1}$. The event, called GW170817, produced gravitational waves, which had weakened to a faint 'chirp' by the time they reached us. In addition, some of the matter in the neutron stars was ejected into space. Moments later, this matter was hit by a powerful jet of material from the merged stars, resulting in a roaring outburst of radiation at all wavelengths ${ }^{2}$. However, despite a flood of data, the process by which this radiation was generated has not been certain. On page 207,

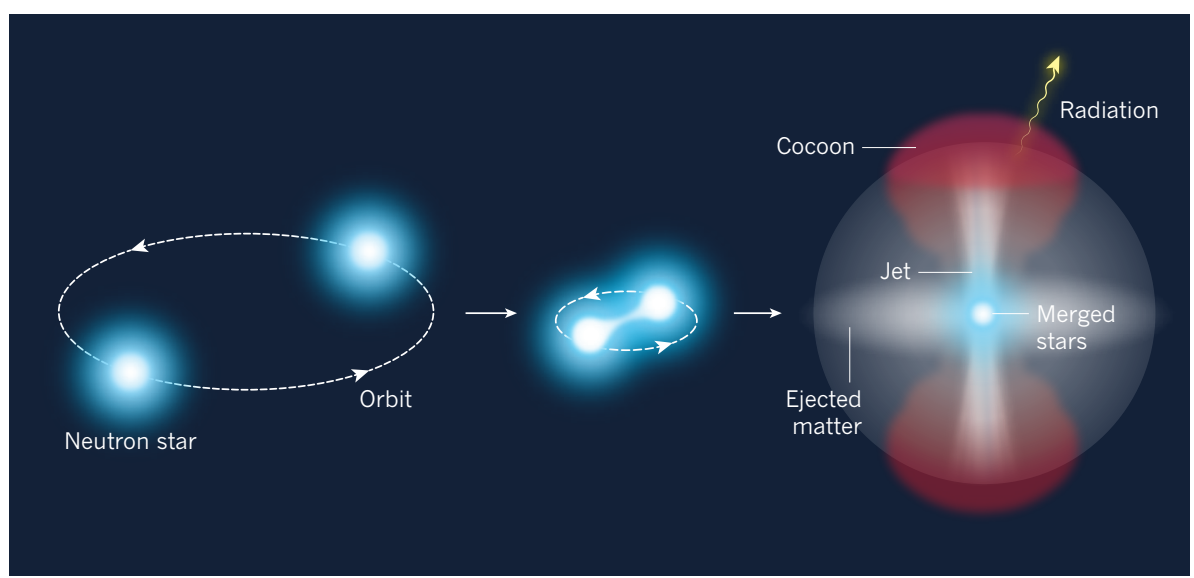

Figure 1 | Radiation from a neutron-star merger. A pair of stellar remnants called neutron stars can orbit each other, gradually getting closer, before eventually merging. In 2017, electromagnetic radiation was detected from a neutron-star merger ${ }^{2}$. Mooley et al. ${ }^{3}$ report evidence for a model that explains how this radiation was generated. In the model, some of the matter in the neutron stars is ejected. This matter is then energized by a powerful jet of material from the merged stars, creating a relatively slow-moving 'cocoon' of matter. The cocoon then emits the observed radiation. fields, nuclear reactions, particle acceleration and radiation come into play. Astronomers cannot create and tune experiments, but must make do with the messy ones performed by nature.

What astronomers can do, however, is take advantage of two of the biggest revolutions in the field since the invention of the telescope. First, in the twentieth century, astronomy became multi-wavelength: we can now detect radiation across the electromagnetic spectrum (from radio waves to $\gamma$-rays). Second, in this century, it became multi-messenger: we can now detect a broad range of emissions from high-energy cosmic rays and neutrinos to gravitational waves. The discovery of GW170817 demonstrated the full potential of these advances for the first time.

After being alerted to the gravitational-wave signal, astronomers used just about every type of telescope available to try to view the event. As a result, a wide variety of data was obtained, potentially providing enough information to pin down a complete picture of what physically happened when the neutron stars merged. In particular, NASA's Fermi Gamma-ray Space Telescope detected a flash of $\gamma$-rays that had formed within two seconds of the merger ${ }^{7}$. The properties of the flash were consistent with a $\gamma$-ray burst (a cosmic explosion long thought to be related to neutron-star mergers), which immediately increased interest in GW 170817. However, the exact cause of the $\gamma$-ray emission became a matter of debate.

Standard $\gamma$-ray bursts can be produced only by a jet - an outflow of material moving at a speed at least $99.9 \%$ that of light. But the burst from GW170817 was about 10,000 times weaker than these bursts and seen only because it occurred relatively close to us ${ }^{7}$. Such a weak burst could have come from an off-axis jet (one that was aimed away from us), which would allow only the tiny fraction of light that it emitted sideways to be observed. But it could also have been produced by a comparatively slowmoving cocoon of matter, perhaps travelling at 'only' 95\% of the speed of light (Fig. 1).

The initial papers ${ }^{2,8}$ concluded that both scenarios are possible, and that additional data should allow us to identify which one is correct. Mooley et al. now fulfil this promise. They show that although the outburst of radiation 
from GW170817 is dying down, the intensity of its radio emission is rising - a finding that is difficult to explain using the relatively simple jet models that they consider. The presence of an off-axis jet that breaks free of the surrounding material is not completely excluded, but the cocoon model is more consistent with the observational data.

Establishing the origin of the electromagnetic emission from GW170817 is key to gaining a detailed understanding of the relationship between gravitational-wave events, neutron-star mergers and $\gamma$-ray bursts. If a consideration of a greater number of jet and cocoon models than that of Mooley et al., and high-quality simulations of these models, support the authors' conclusions, nature will once again have shown us that the range of phenomena possible is wider than our simplest thinking suggested. If the cocoon model is correct, this probably implies that many more gravitational-wave events have associated $\gamma$-ray bursts than was previously thought.

However, Mooley and colleagues' explanation for the burst does not affect our basic understanding of what happens in a neutronstar merger - all of the models considered by the authors have great commonality. For instance, the merged stars are always surrounded by matter that is both inflowing (in the equatorial plane of the merged stars) and outflowing (in all other directions). And a faster and narrower outflow is always driven into this matter along the rotational axis of the merged stars.

The differences are in the precise outcome of the attendant fluid dynamics. How much mass is contained in slow parts of the surrounding matter, and how fast and narrow is the jet? Does the jet break out of the surrounding matter so that it can be seen by us, or does it share its energy with this material, producing a relatively slow and broad explosion?

The next few years will see fierce efforts to address these questions. Gravitational-wave events will be under surveillance by an army of telescopes, to find or exclude a fast jet. And elaborate computer simulations will be used to try to determine what we should expect to happen in neutron-star mergers from a theoretical standpoint. The next handful of well-observed events will bring us much closer to the answers.

Ralph Wijers is at the Anton Pannekoek Institute for Astronomy, Faculty of Science, University of Amsterdam, $1098 \mathrm{XH}$

Amsterdam, the Netherlands. e-mail:ralph.wijers@uva.nl

1. Abbott, B. P. et al. (LIGO Scientific Collaboration and Virgo Collaboration) Phys. Rev. Lett. 119, 161101 (2017).

2. Abbott, B. P. et al. Astrophys. J. 848, L12 (2017).

3. Mooley, K. P. et al. Nature 554, 207-210 (2018).

4. Hulse, R. A. \& Taylor, J. H. Astrophys. J. 195, L51-L53 (1975).

5. Taylor, J. H. \& Weisberg, J. M. Astrophys. J. 253,
6. Abbott, B. P. et al. (LIGO Scientific Collaboration and Virgo Collaboration) Phys. Rev. Lett. 116 061102 (2016).

7. Goldstein, A. et al. Astrophys. J. 848, L14 (2017).

8. Kasliwal, M. M. et al. Science 358, 1559-1565 (2017).

\title{
OPTICAL PHYSICS
}

\section{Solitons divide and conquer}

\begin{abstract}
An experimental technique allows packets of light called solitons to maintain their shape in all three dimensions as they travel through a material. Such wave packets could find applications in optical information processing.
\end{abstract}

\section{FRANK W. WISE}

$\mathrm{W}$ aves spread out as they propagate. A familiar example is the broadening of a beam of light. The challenge of overcoming the ubiquitous spreading of waves has motivated scientists for decades, and packets of light waves that retain their shape, known as solitons, have been demonstrated in one and two dimensions ${ }^{1}$. However, it has been extremely difficult to create solitons that are stable in three dimensions. Writing in Physical Review X, Lahav et al. ${ }^{2}$ report an experimental approach that can produce such objects. The work will allow fundamental properties of $3 \mathrm{D}$ solitons to be investigated, and could lead to 3D solitons that have technological applications.

A narrow beam of light contains wave components that propagate in different directions. As the beam travels through a material, these wave components get out of sync, causing the beam to spread out - a process known as diffraction. However, if the beam is powerful enough, the light changes the material's refractive index (a quantity that describes how light propagates in a medium), which in turn affects the beam. In particular, if the beam has a bell-shaped intensity profile, as do most laser beams, the material focuses the beam like a lens. By tuning the beam intensity, this focusing can counteract diffraction to produce a 'self-guided' beam that does not spread out.

In addition to diffraction, a pulsed beam exhibits a broadening effect along its direction of propagation. Each pulse of light contains wave components that have a range of frequencies (colours), and, as a pulse moves

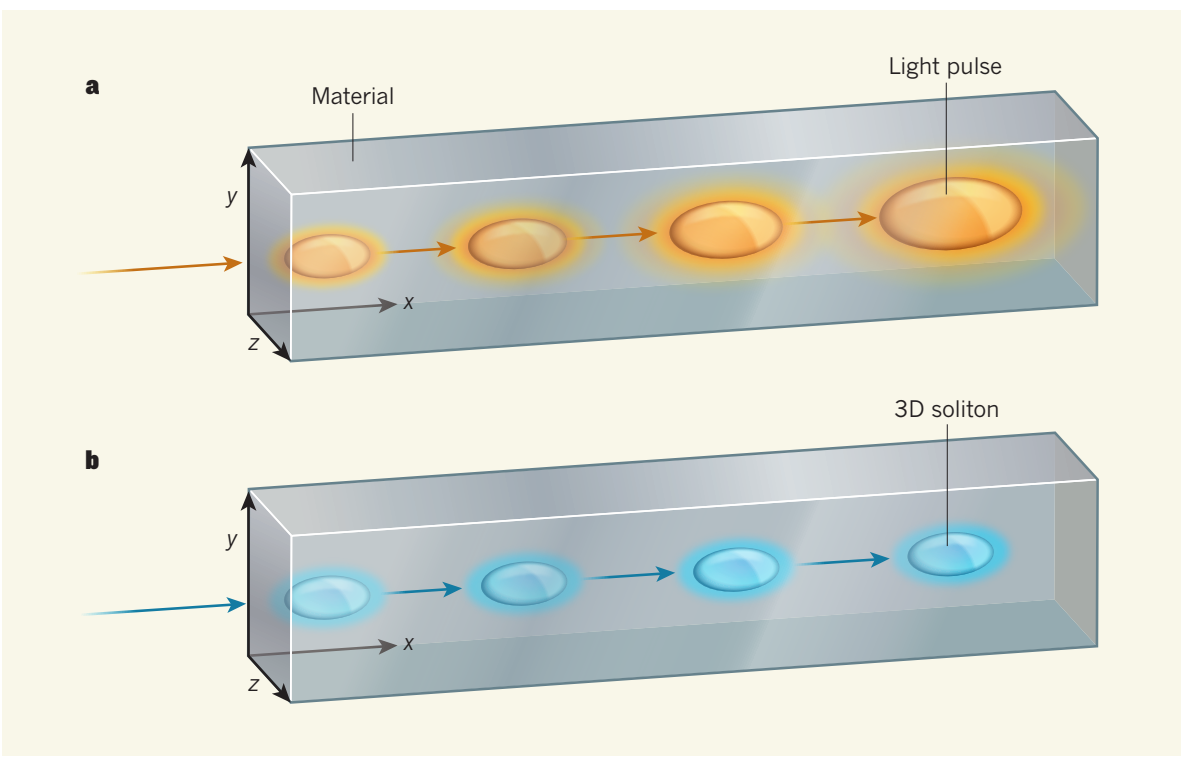

Figure 1 | Light pulses versus 3D solitons. a, A pulse of light tends to spread out as it propagates through a material (coloured arrows). It broadens along the direction in which it is travelling (the $x$ axis) as a result of dispersion, whereby components of the pulse that have different frequencies separate. Furthermore, it widens along the perpendicular directions (the $y$ and $z$ axes) because of diffraction. $\mathbf{b}$, Lahav et al. ${ }^{2}$ report a technique for producing three-dimensional solitons - packets of light that maintain their geometry as they move through a material. 